\title{
Rechallenge of oxaliplatin-containing regimens in the third- or later-line therapy for patients with heavily treated metastatic colorectal cancer
}

This article was published in the following Dove Press journal:

OncoTargets and Therapy

\author{
Qiong Yang ${ }^{1-3, *}$ \\ Yuanyuan Huang ${ }^{4-6, *}$ \\ Zhimin Jiang ${ }^{1-3}$ \\ Huizhong Wang ${ }^{1-3}$ \\ Weiyu $\mathrm{Li}^{1-3}$ \\ Bei Zhang ${ }^{4-6}$ \\ Derong $\mathrm{Xie}^{1-3}$ \\ 'Department of Oncology, Sun \\ Yat-sen Memorial Hospital, Sun \\ Yat-sen University, Guangzhou, \\ China; ${ }^{2}$ Guangdong Provincial Key \\ Laboratory of Malignant Tumor \\ Epigenetics and Gene Regulation, \\ Sun Yat-sen Memorial Hospital, Sun \\ Yat-sen University, Guangzhou, \\ China; ${ }^{3}$ Medical Research Center, \\ Sun Yat-sen Memorial Hospital, Sun \\ Yat-sen University, Guangzhou, China; \\ ${ }^{4}$ VIP Region, Sun Yat-sen University \\ Cancer Center, Guangzhou, China; \\ ${ }^{5}$ State Key Laboratory of Oncology in \\ South China, Sun Yat-sen University \\ Cancer Center, Guangzhou, China; \\ ${ }^{6}$ Collaborative Innovation Center for \\ Cancer Medicine, Guangzhou, China \\ *These authors contributed equally \\ to this work
}

Correspondence: Derong Xie Department of Oncology, Sun Yat-sen Memorial Hospital, Sun Yat-sen University, 107 Yangjiang Road West, Guangzhou 510120, China

Tel +862081332616

Fax +86 2087343392

Email derongxie@|26.com

Bei Zhang

VIP Region, Sun Yat-sen University

Cancer Center, 65I Dongfeng Road East,

Guangzhou 510060, China

Tel +862087343455

Fax +86 2087343392

Email zhangbei@sysucc.org.cn
Purpose: The third- or later-line therapy available often yield poor survival benefit in patients metastatic colorectal cancer ( $\mathrm{mCRC}$ ). The retrospective study aimed to evaluate efficacy of rechallenge of oxaliplatin-containing regimens.

Patients and methods: Patients with $\mathrm{mCRC}$ who progressed from fluoropyrimidine, oxaliplatin, and irinotecan in the first- and second-line chemotherapy, were treated by reexposure to oxaliplatin-containing regimen. Patients treated by anti-epidermal growth factor receptor (EGFR) antibodies with irinotecan were included in the control arm.

Results: Ninety-five and 29 patients were treated with either oxaliplatin reexposure or antiEGFR antibodies with irinotecan, respectively, as the third- or later-line therapy. The median time to treatment failure (TTF) and overall survival (OS) was 3.77 and 12.17 months in the oxaliplatin arm, with 4.77 months of TTF and 11.37 months of OS in the control arm; there was no significance between the 2 arms $(p>0.05)$. Oxaliplatin reexposure resulted in $6.3 \%$ objective response rate with no complete response, 6 partial response, 39 stable disease, and 37 progressive disease. The disease control rate was $47.4 \%$ (45/95). The multivariate analysis found that patients who achieved disease control by oxaliplatin reexposure had a superior TTF (6.13 vs 1.7 months, $p<0.001)$ and OS (15.73 vs 6.27 months, $p<0.001)$ compared with those presenting with progressive disease.

Conclusion: This study showed that rechallenge of oxaliplatin-containing chemotherapy in the third- or later-line therapy may lead to tumor control and improved survival in mCRC patients, which was equivalent to that of anti-EGFR antibodies with irinotecan.

Clinical significance: Rechallenge of oxaliplatin-containing regimens in the third- or later-line of therapy is a common practice, despite few evidence available. The present study found that rechallenge of oxaliplatin-containing regimens produced equivalent tumor control and survival benefit to that of anti-EGFR antibodies with irinotecan in mCRC.

Keywords: rechallenge, oxaliplatin, colorectal cancer, anti-epidermal growth factor receptor

\section{Introduction}

Colorectal cancer ranks as the third most commonly diagnosed cancer in males and the second in females worldwide, with 1,400,000 new cases and 693,900 deaths estimated to have occurred in $2012 .{ }^{1}$ Among newly diagnosed cases, $\sim 40 \%-50 \%$ of them have metastatic disease. ${ }^{2}$ Chemotherapy alone or in combination with monoclonal antibodies has proven survival benefit and has been accepted as the standard first- or second-line treatment for metastatic colorectal cancer (mCRC). ${ }^{3,4}$ The overall survival (OS) is reported to be about 30 months in average. ${ }^{5}$ However, more than one-thirds of patients with $\mathrm{mCRC}$ still have a chance to receive 3 or more lines of therapy. ${ }^{6,7}$ 
Recently, at least 4 regimens are recommended by National Comprehensive Cancer Network guideline as the third- or later-line therapy, which include anti-epidermal growth factor receptor (anti-EGFR) monoclonal antibodies with or without irinotecan (Pfizer, New York, USA), regorafenib (Bayer, Leverkusen, German), TAS-102 (Taiho Oncology Inc, NJ, USA), and programmed cell death protein-1 (PD-1) blockade. ${ }^{8}$ Several trials have documented that anti-EGFR monoclonal antibodies with or without irinotecan improved antitumor activity and OS in patients with irinotecanrefractory colorectal cancer, with objective response rate (ORR) of 20\%-25.7\%, time to progression (TTP) ranging from 3.4 to 5.4 months, and OS of 8.0-10.4 months. ${ }^{9,10}$ The CORRECT trial reported that regorafenib might add survival benefit in patients with $\mathrm{mCRC}$, with an ORR of $1 \%$, 1.9-month progression-free survival (PFS) and an OS of 6.4 months. ${ }^{2}$ In the RECOURSE trial, mCRC patients with TAS-102 who progressed through at least 2 previous regimens showed superior efficacy compared with the placebo group in PFS (2.0 months vs 1.7 months, $p<0.001)$ and OS (7.1 months vs 5.3 months, $p<0.001) .{ }^{11}$ A Phase II study evaluated clinical activity of pembrolizumab for treatment of patients with DNA mismatch repair deficiency (dMMR) $\mathrm{mCRC}$ and who had progressed through 2 or more therapies and found that the immune-related ORR was $40 \%$ and the immune-related PFS rate at 20 weeks was $78 \%{ }^{12}$

Despite survival benefits, the aforementioned regimens are not widely adopted in clinical practice possibly due to the following reasons. First, the treatments only benefit a limited number of patients. For example, about $40 \%$ of mCRC patients have wild-type RAS and meet the eligibility for therapies using anti-EGFR antibodies. ${ }^{12}$ Second, dMMR is rare in mCRC and $3.5 \%-5.0 \%$ of mCRC may benefit from PD-1 blockade. ${ }^{13}$ Third, the absolute survival benefit of both tyrosine kinase inhibitors and TAS-102 is very limited. ${ }^{2,11}$ Finally, but importantly, the 4 recommended regimens are very expensive and not all patients can afford them in China. Considering these obstacles, further efforts are needed to explore more cost-effective regimens for patients in China.

According to our clinical experience, patients who have progressed from standard therapies and have a good performance are suggested to continue oxaliplatin (Sanofi-Aventis, Paris, France)-containing regimens as the later-line therapy. However, limited data is available on its clinical efficacy. In the present retrospective study, we aimed to evaluate the efficacy of rechallenge of oxaliplatin-containing regimens in Chinese mCRC patients who have failed 2 or more lines of standard therapy.

\section{Patients and methods}

\section{Patients}

Between April 2004 and October 2013, we retrospectively reviewed 1,140 histologically confirmed and measurable stage IV CRC patients at the Sun Yat-sen University Cancer Center. The major selection criterion was receipt of oxaliplatincontaining chemotherapy in the third- or later-line therapies. Other criteria for eligibility were as follows: 1) prior use of oxaliplatin as the first- or second-line therapy, 2) Eastern Cooperative Oncology Group (ECOG) performance scores of $0-2,3$ ) adequate hepatic function bilirubin $<2.0 \mathrm{mg} / \mathrm{dL}$, transaminases levels $<3$ times the upper normal limit ( 5 times for patients with liver metastasis), 4) adequate renal function (creatinine $<1.5 \mathrm{mg} / \mathrm{dL}$ ), 5) adequate bone marrow function (absolute neutrophil count $>1,500 / \mu \mathrm{L}$, hemoglobin $>9.0 \mathrm{~g} / \mathrm{dL}$, and platelets $>75,000 / \mu \mathrm{L}$ ), and 6) expected life expectancy of $>3$ months. Written informed consent was required before chemotherapy.

Exclusion criteria included 1) peripheral neuropathy $>$ grade 1 as measured by NCI-CTCAE $4.0 ; 2$ ) disease relapse after the termination of oxaliplatin treatments within 6 months; 3) resumption of treatment after an oxaliplatin-free interval because of toxicity; and 4) no clinical data available obtained.

In the control group, patients were treated with anti-EGFR antibodies with irinotecan in later-line therapy.

This retrospective study was approved by the Institutional Review Board of the Sun Yat-sen cancer center. All inpatients were informed that their medical records may be reviewed for scientific research purposes and their individual confidentiality would be protected in accordance with the ethical standards of the Declaration of Helsinki; therefore, no written informed consent was required for this research.

\section{Statistical analyses}

Descriptive statistics were reported as proportion and median. Tumor responses were assessed by RECIST 1.1 criteria every 6-8 weeks. Radiologic evaluation consisted of chest and abdominopelvic CTs. Time to treatment failure (TTF) was defined as the time from the initial of oxaliplatin rechallenge or anti-EGFR antibodies with irinotecan in the third- or later-line treatment to treatment disruption for the following reasons: disease progression, treatment toxicity, patient preference, surgery, or death. OS was defined as the time from the initial of oxaliplatin rechallenge or anti-EGFR antibodies with irinotecan in the third- or later-line treatment to death from any cause or loss of follow-up. Fisher's exact test and $\chi^{2}$ analysis 
were used, as appropriate, for categorical data. TTF or OS was calculated using the Kaplan-Meier method and data were compared using the log-rank test. All factors possibly influencing TTF or OS of oxaliplatin rechallenge were evaluated using univariate and, subsequently, multivariate analyses. Two-tailed $p$-values of $<0.05$ were considered statistically significant. SPSS version 18.0 was used for statistical analysis.

\section{Results \\ Patient characteristics}

Between April 2004 and October 2013, 95 out of 1,140 consecutive patients with $\mathrm{mCRC}$ were retreated with oxaliplatincontaining regimens and included in the final analyses. Meanwhile, 29 patients treated with anti-EGFR antibodies with irinotecan served as the control arm.

In the oxaliplatin arm $(n=95)$, the median age of patients was 54 years old (range: $22-79$ years). The majority of patients were male $(63.2 \%)$ and had left-sided colorectal cancer (73.7\%). Kirsten rat sarcoma viral oncogene (KRAS) was detected in 59 patients, and which showed 45 of wild type and 14 of mutated type. In total, 28 patients were diagnosed with stage I-III and predominantly received adjuvant oxaliplatin chemotherapy. At the first time of detection of recurrent or metastatic disease, 25 patients $(26.3 \%)$ had only liver metastases. Eighty percent of patients $(n=76)$ who were exposed to $1-20$ cycles of first oxaliplatin treatment as the first-line therapy had a median of 10.18 months of PFS. The remaining patients who received 2-12 cycles of first oxaliplatin treatment in the second-line therapy had a median PFS of 10.03 months. The oxaliplatin-containing chemotherapy re-treatment regimens were mainly 5-fluorouracil, levoleucovorin, and oxaliplatin $(\mathrm{mFOLFOX})(\mathrm{n}=70,73.7 \%)$ and capecitabine and oxaliplatin (XELOX) $(n=19,20 \%)$. The treatment schedules in terms of dosage, number of cycles, and the interval between cycles were strictly followed against guideline. Furthermore, 45 patients had received anti-vascular endothelial growth factor (anti-VEGF) - or anti-EGFR-containing therapy. The proportions of patients retreated with oxaliplatin in the third, fourth, and later line were $82.1 \%, 13.7 \%$, and $4.2 \%$, respectively.

For the irinotecan arm, the demographic and clinical characteristics were comparable with the oxaliplatin arm, except for having a higher percentage of patients classified as wild-type mCRC (58.6\% vs $47.4 \%$ ) (Table 1$)$.

\section{Efficacy}

Until July 18, 2017, the median follow-up time from disease recurrence/metastasis was 33 months (range: 6.7-118.8
Table I Baseline patient characteristics

\begin{tabular}{|c|c|c|}
\hline \multirow[t]{2}{*}{ Variables } & \multicolumn{2}{|c|}{ No. patients (\%) } \\
\hline & $\begin{array}{l}\text { Rechallenge } \\
\text { of oxaliplatin }\end{array}$ & $\begin{array}{l}\text { Anti-EGFR } \\
\text { antibodies with } \\
\text { irinotecan }\end{array}$ \\
\hline \multicolumn{3}{|l|}{ Age, years } \\
\hline Median (range) & $54(22-79)$ & $53(26-87)$ \\
\hline \multicolumn{3}{|l|}{ Sex } \\
\hline Female & $35(36.8)$ & $21(72.4)$ \\
\hline Male & $60(63.2)$ & $8(27.6)$ \\
\hline \multicolumn{3}{|l|}{ Primary tumor } \\
\hline Left-sided & $70(73.7)$ & $21(72.4)$ \\
\hline Right-sided & $25(26.3)$ & $8(27.6)$ \\
\hline \multicolumn{3}{|l|}{ Initial stage } \\
\hline I & $2(2.1)$ & $0(0)$ \\
\hline ॥ & $9(9.5)$ & I (3.4) \\
\hline III & $17(17.9)$ & $6(20.7)$ \\
\hline IV & $67(70.5)$ & $22(75.9)$ \\
\hline \multicolumn{3}{|l|}{ KRAS status } \\
\hline Wild type & $45(47.4)$ & $17(58.6)$ \\
\hline Mutated type & $14(14.7)$ & I (3.4) \\
\hline Unknown & $36(37.9)$ & II (37.9) \\
\hline \multicolumn{3}{|l|}{ Sites of metastases } \\
\hline Liver & $25(26.3)$ & $6(20.7)$ \\
\hline Other & $70(73.7)$ & $23(79.3)$ \\
\hline \multicolumn{3}{|c|}{ Adjuvant therapy with oxaliplatin } \\
\hline Yes & $21(22.1)$ & $6(20.7)$ \\
\hline No & $7(7.4)$ & I (3.4) \\
\hline \multicolumn{3}{|c|}{ First oxaliplatin as line number } \\
\hline First & $76(80.0)$ & ND \\
\hline Second & $19(20.0)$ & ND \\
\hline \multicolumn{3}{|c|}{ Chemotherapy regimen of rechallenge of oxaliplatin } \\
\hline mFOLFOX6 & $70(73.7)$ & ND \\
\hline XELOX & $19(20)$ & ND \\
\hline Other & $6(6.3)$ & ND \\
\hline \multicolumn{3}{|c|}{ Monoclonal antibodies } \\
\hline Bevacizumab & $34(35.8)$ & ND \\
\hline Cetuximab & II (II.6) & ND \\
\hline None & $50(52.6)$ & ND \\
\hline \multicolumn{3}{|c|}{ Rechallenge of oxaliplatin as line number } \\
\hline Third & $78(82.1)$ & ND \\
\hline Fourth & $13(13.7)$ & ND \\
\hline Fifth or more & $4(4.2)$ & ND \\
\hline \multicolumn{3}{|c|}{ Number of rechallenge of oxaliplatin cycles } \\
\hline Median (range) & $3(1-12)$ & ND \\
\hline
\end{tabular}

Abbreviations: EGFR, epidermal growth factor receptor; KRAS, Kirsten rat sarcoma viral oncogene; mFOLFOX6, 5-fluorouracil, levoleucovorin, and oxaliplatin; ND, no data; XELOX, capecitabine and oxaliplatin.

months). In the oxaliplatin arm $(n=95), 84.2 \%$ and $83.2 \%$ of patients had experienced progression and died at the last follow-up, respectively. The median number of cycles of oxaliplatin reexposure was 3 (range: 1-12). The primary reason for discontinuation of treatment was disease progression $(84.2 \%)$, and the other reasons included patient refusal $(n=9,9.5 \%)$, inability to tolerate treatment $(n=3,3.2 \%)$, 
palliative surgery $(n=1,1.1 \%)$, or death $(n=1,1.1 \%)$. For the control arm $(n=29), 82.8 \%$ and $89.7 \%$ of patients had experienced progression and died at the last follow-up, respectively. The median number of cycles of anti-EGFR antibodies with irinotecan completed was 3 (range: $1-18$ ). In addition to disease progression $(n=26), 3$ patients discontinued treatment due to treatment toxicity. After progression from rechallenge of oxaliplatin, 45 (47.4\%) patients received later treatments, in rechallenge of oxaliplatin arm, of which 11 patients were treated with anti-EGFR antibody monotherapy or combination with irinotecan, 6 with famitinib, 4 with fruquintinib, 1 with gemcitabine, and the others with fluoropyrimidinebased regimens. In anti-EFGR antibodies with irinotecan arm, $12(43.4 \%)$ patients received post-treatment after progression from anti-EGFR antibodies with irinotecan, and of which 7 patients treated with oxaliplatin-containing regimens, 2 patients treated with famitinib, and the other three treated with panitumumab, TAS102 and pemetrexed, respectively. The overall post-treatments between the 2 arms were similar $(p=0.57)$.

In the oxaliplatin arm, the ORR was $6.3 \%$ with no complete response (CR), 6 (6.3\%) of partial response (PR), $39(41.1 \%)$ of stable disease (SD), and 37 (38.9\%) of progressive disease (PD). The remaining 13 patients were not evaluated. The disease control rate (DCR) was 47.4\% (45/95; Table 2). The median TTF from rechallenge of oxaliplatin was 3.77 months (95\% CI: 3.00-4.54), while the median OS was 12.17 months (95\% CI: 10.85-13.48; Figures 1 and 2). In the irinotecan arm, the ORR was $13.8 \%$ with 4 PR. The DCR was $51.7 \%(15 / 29)$. The median TTF and OS were 4.77 months (95\% CI: 1.33-8.20) and 11.37 months $(95 \%$ CI: 8.26-14.48), respectively. The TTF and OS were not different between the oxaliplatin and irinotecan arms according to the Kaplan-Meier curve analysis ( $p=0.90, p=0.80$, respectively; Figures 1 and 2). The $\chi^{2}$ test indicated insignificant results for ORR between 2 arms ( $p=0.28$; Table 2). In the univariate analyses for rechallenge of oxaliplatin, the patient's age, sex, stage and location of primary tumor, sites

Table 2 Tumor response and survival

\begin{tabular}{llll}
\hline & $\begin{array}{l}\text { Rechallenge } \\
\text { of oxaliplatin }\end{array}$ & $\begin{array}{l}\text { Anti-EGFR } \\
\text { antibodies + CPT- I I }\end{array}$ & p-value \\
\hline RR & $6.32 \%$ & $13.80 \%$ & 0.28 \\
DCR & $47.40 \%$ & $51.70 \%$ & 0.46 \\
Median & $3.77,95 \% \mathrm{Cl}:$ & $4.77,95 \% \mathrm{Cl}:$ & 0.90 \\
TTF, mos & $3.00-4.54$ & $1.33-8.20$ & 0.80 \\
Median & $12.17,95 \% \mathrm{Cl}:$ & $11.37,95 \% \mathrm{Cl}:$ & \\
OS, mos & $10.85-13.48$ & $8.26-14.48$ & \\
\hline
\end{tabular}

Abbreviations: CPT-II, irinotecan; DCR, disease control rate; EGFR, epidermal growth factor receptor; mos, months; OS, overall survival; RR, response rate; TTF, time-to-treatment failure.

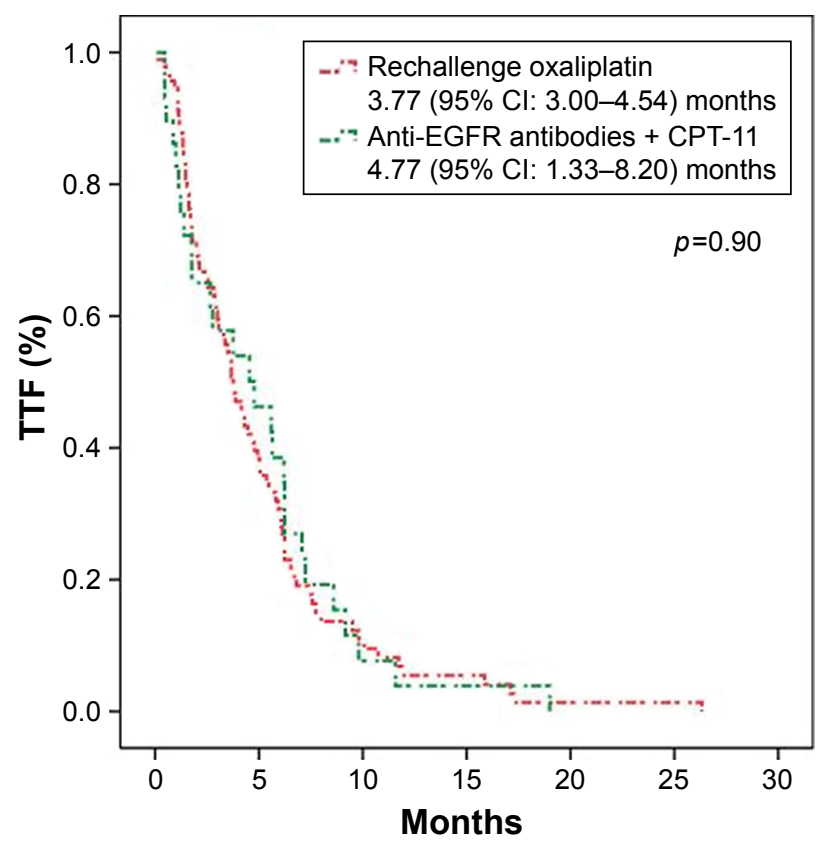

Figure I Kaplan-Meier estimates of TTF from start of oxaliplatin rechallenge and anti-EGFR antibodies with irinotecan.

Abbreviations: EGFR, epidermal growth factor receptor; TTF, time-to-treatment failure.

of metastases, status of KRAS mutation, monoclonal antibodies treatment, reexposure to oxaliplatin, lines of rechallenge of oxaliplatin, and the best response to the first oxaliplatin were not associated with TTF $(p>0.05)$. Only patients who achieved disease control (DC) by reexposure to oxaliplatin had a superior TTF when compared with those presented

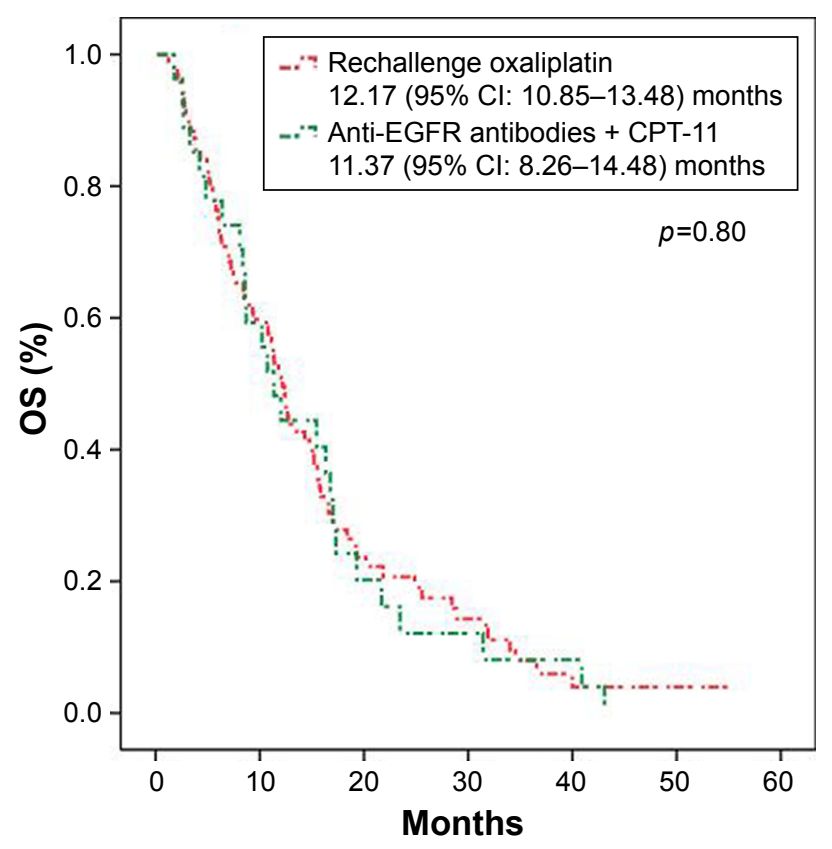

Figure 2 Kaplan-Meier estimates of OS from initial of oxaliplatin rechallenge and anti-EGFR antibodies with irinotecan.

Abbreviations: EGFR, epidermal growth factor receptor; OS, overall survival. 
with PD (6.13 vs 1.7 months, $p<0.001)$. Correlation analysis was further conducted to explore the possible influence of the best response to the first oxaliplatin on efficacy of oxaliplatin reexposure, but there was no statistically significant correlation identified $(p=0.81)$. In the univariate analyses, patients who were male or achieved DC disease were found to have superior OS from oxaliplatin reexposure, but only DC disease was identified as the independent prognostic factor of OS in the multivariate analysis (DC vs PD: 15.73 months vs 6.27 months, $p<0.001)$. The detailed results are shown in Table 3 and Figures 3 and 4.

Table 3 Univariate and multivariate analyses of factors influencing survival in patients treated by oxaliplatin rechallenge

\begin{tabular}{|c|c|c|c|c|}
\hline Variables & $\begin{array}{l}\text { Median } \\
\text { TTF, mos }\end{array}$ & $p$-value & $\begin{array}{l}\text { Median } \\
\text { OS, mos }\end{array}$ & p-value \\
\hline Age, years & & 0.81 & & 0.34 \\
\hline$<60$ & 3.77 & & 12.43 & \\
\hline$\geq 60$ & 4.17 & & 12.07 & \\
\hline Sex & & 0.12 & & 0.02 \\
\hline Female & 3.37 & & 9.3 & \\
\hline Male & 4.3 & & 14.3 & \\
\hline \multicolumn{2}{|l|}{ Location of primary tumor } & 0.33 & & 0.36 \\
\hline Left-sided & 4.17 & & 12.67 & \\
\hline Right-sided & 2.13 & & 7.23 & \\
\hline Initial stage & & 0.65 & & 0.06 \\
\hline$|/| I / I I I$ & 5.03 & & 18.4 & \\
\hline IV & 3.67 & & 11.27 & \\
\hline KRAS status & & 0.30 & & 0.82 \\
\hline Wild type & 4.17 & & 12.07 & \\
\hline Mutated type & 2.1 & & 16.6 & \\
\hline Unknown & 4.17 & & 12.17 & \\
\hline Sites of metastases & & 0.78 & & 0.92 \\
\hline Liver & 3.3 & & 12.53 & \\
\hline Others & 3.83 & & 12.07 & \\
\hline Monoclonal antibodies & & 0.37 & & 0.50 \\
\hline Yes & 4.47 & & 14.3 & \\
\hline No & 3.67 & & 11.13 & \\
\hline \multicolumn{2}{|c|}{$\begin{array}{l}\text { Regimens of rechallenge of } \\
\text { oxaliplatin }\end{array}$} & 0.61 & & 0.60 \\
\hline mFOLFOX6 & 3.87 & & 11.13 & \\
\hline XELOX & 3.07 & & 12.67 & \\
\hline Other & 2 & & 15.83 & \\
\hline \multicolumn{2}{|c|}{ Line of rechallenge of oxaliplatin } & 0.60 & & 0.41 \\
\hline Third line & 3.87 & & 12.43 & \\
\hline Fourth line or more & 3.5 & & 8.57 & \\
\hline \multicolumn{2}{|c|}{ Best response to first oxaliplatin } & 0.27 & & 0.75 \\
\hline DC & 4.3 & & 11.37 & \\
\hline PD & 2.9 & & 14.3 & \\
\hline \multicolumn{2}{|c|}{$\begin{array}{l}\text { Best response to rechallenge of } \\
\text { oxaliplatin }\end{array}$} & 0.00 & & 0.00 \\
\hline DC & 6.13 & & 15.73 & \\
\hline PD & 1.7 & & 6.27 & \\
\hline
\end{tabular}

Abbreviations: DC, disease control; KRAS, Kirsten rat sarcoma viral oncogene; mFOLFOX6, 5-fluorouracil, levoleucovorin, and oxaliplatin; mos, months; OS, overall survival; PD, progression of disease; TTF, time-to-treatment failure; XELOX, capecitabine and oxaliplatin.

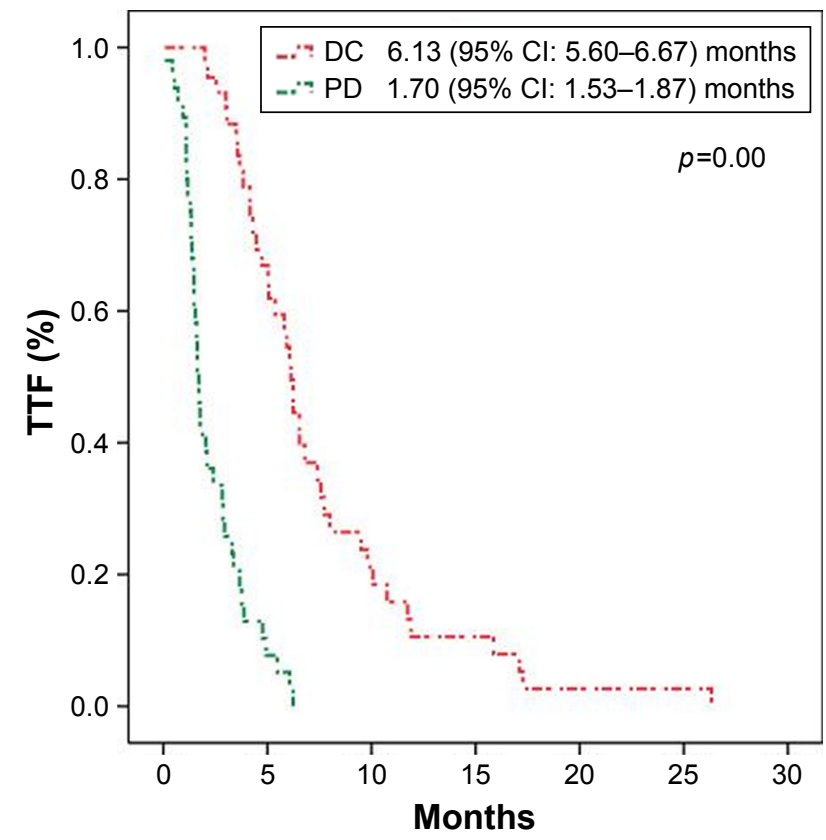

Figure 3 Subgroup analysis of TTF based on best response to oxaliplatin rechallenge. Abbreviations: DC, disease control; PD, progressive disease; TTF, time-totreatment failure.

\section{Discussion}

Previous studies reported that about $34 \%-43.2 \%$ of the patients with $\mathrm{mCRC}$ who progressed after receiving all standard therapies had received third- or later-line treatment. ${ }^{6,7}$ Although there are alternative later-line therapies available, the efficacy of these treatments has been discouraging. Consistent with previous reports, our study found that

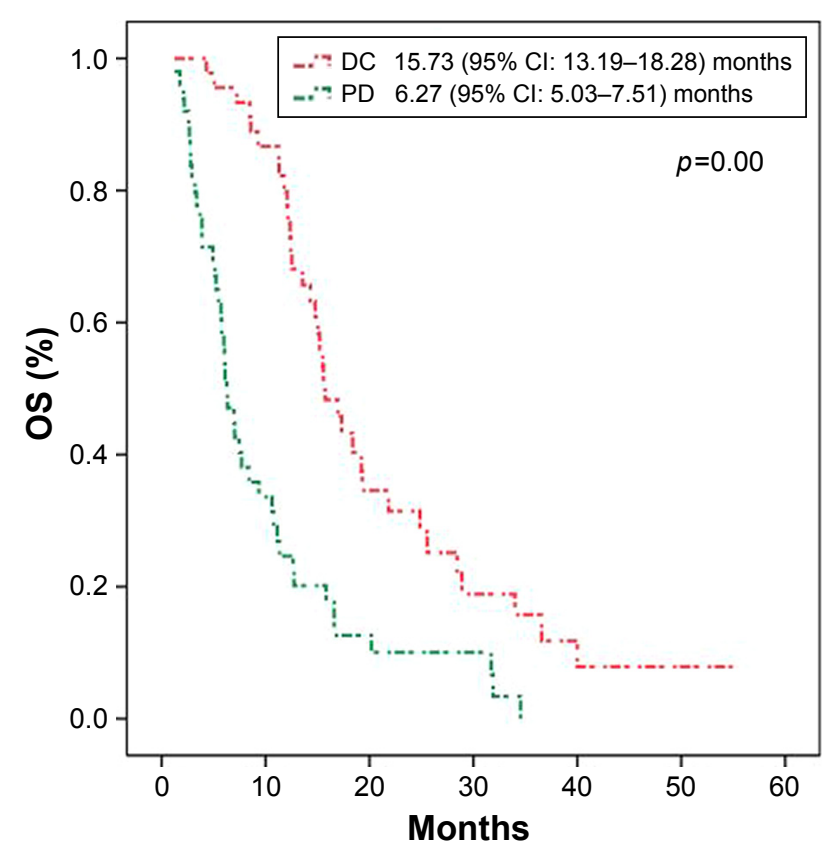

Figure 4 Subgroup analysis of OS based on best response to oxaliplatin rechallenge. Abbreviations: DC, disease control; PD, progressive disease; OS, overall survival. 
anti-EGFR antibodies with irinotecan resulted in a ORR of $13.8 \%$, a median TTF of 4.77 months and a median OS of 11.37 months in the control arm, ${ }^{14,15}$ However, in the realworld practice, mCRC patients after experiencing primary treatment failure, especially with KRAS mutated type, require reexposure to oxaliplatin-containing regimens. ${ }^{16}$

In this study, reexposure to an oxaliplatin-containing regimen yielded an ORR of $6.3 \%$, a median TTF of 3.77 months, and a median OS of 12.17 months. There were no significant differences in treatment efficacy between patients treated with oxaliplatin and anti-EGFR antibodies with irinotecan. This may suggest that reexposure to oxaliplatin-containing chemotherapy might be an alternative option as a later-line treatment for patients with $\mathrm{mCRC}$.

Very limited studies exist on examining the role of reexposure to oxaliplatin in third- or later-line therapy in mCRC patients. In a smaller Italian Phase II study $(n=12),{ }^{17}$ the reexposure to oxaliplatin resulted in a PR and SD in $25 \%$ and $50 \%$ of patients, respectively. However, this study did not report any treatment efficacy data on the PFS and OS. Another Japanese study of mCRC patients previously treated with oxaliplatin and irinotecan found that reexposure to oxaliplatin produced $6.1 \%$ ORR, a median PFS of 3.2 months, and a median OS of 10 months. ${ }^{18}$ However, this study is limited by the small sample size and lack of a control arm. A retrospective study reported similar results in terms of $56 \%$ of DCR, 6.04 months of TTF, and 10.4 months of OS following rechallenging of oxaliplatin. ${ }^{16}$ It was noteworthy that this study included patients who were not reexposed to oxaliplatin as the third and later line and had no data on ORR. ${ }^{16}$ In addition, other studies reported $6.3 \%-36 \%$ of ORR, 4.4-8 months of PFS/TTP/TTF, and 6-16 months of OS. ${ }^{19-22}$ However, these studies are limited by the fact that not all patients received oxaliplatin during the first- and/or second-line treatment. To sum up, our data suggest that the rechallenge of oxaliplatin in the third- or later-line therapy may lead to better tumor control and improved survival benefits for mCRC patients.

In the Japanese study, multivariate analysis showed that good performance status, a single site metastasis, and disease control were independent determinants of longer PFS. In the case of OS, being male and single site metastasis were found to be positive predictors of OS. ${ }^{18}$ In a retrospective study, patients who attained disease control had higher median OS (14.5 vs 6.24 months; $p<0.001$ ) and TTF (7.5 vs 3.6 months; $p<0.001$ ) when compared with those with PD. ${ }^{16}$ Similarly, in our study, patients who attained DC disease after rechallenge of oxaliplatin had a longer TTF (6.13 vs 1.7 months; $p=0.00$ ) and OS (15.73 vs 6.27 months; $p=0.00)$. Being of male sex was also found to be correlated with longer OS. Although patients achieving PR or CR by the first oxaliplatin treatment had a longer OS by reexposure to oxaliplatin in the Costa's retrospective study, ${ }^{16}$ our study did not confirm such finding. The 3 studies documented that response to reexposure to oxaliplatin would translate to survival benefit. But progression from the first oxaliplatin doesn't always predict the no response to that of oxaliplatin reexposure. In other words, it is possible that oxaliplatin resistance is transient in $\mathrm{mCRC}$. There is a case report showing possible interaction of oxaliplatin and cetuximab, in patients retreated with oxaliplatin and cetuximab. Bevacizumab is thought to normalize the tumor vessels and to decrease the intratumoral tissue pressure, making the drug influx more effective. ${ }^{23,24}$ In our study, $47.4 \%$ of patients received cetuximab or bevacizumab in combination with oxaliplatin.

\section{Strengths and limitations}

Although the advantages of this study lie in a large sample size and the use of control arm, several limitations may hamper the generalization of the results to larger population. First, selection bias cannot be excluded from the study. We only included patients treated with oxaliplatin-containing third- or later-line therapy but without peripheral sensory neuropathy. The literature documents that about $84 \%$ patients experience long-term peripheral neurotoxicity of up to 2 years following completion of the first cycle of oxaliplation-containing regimen. ${ }^{25}$ Second, the treatment toxicity, especially peripheral neuropathy of oxaliplatin-containing regimen in later-line therapy, was not analyzed because of insufficient data. Finally, due to the retrospective nature of the study, the endpoint TTF instead of the PFS was used to evaluate treatment benefit, which might not cover the true treatment efficacy of reexposure to oxaliplatin.

\section{Conclusion}

This retrospective study showed that oxaliplatin-containing chemotherapy as third- or later-line therapy may lead to tumor control and improved survival in $\mathrm{mCRC}$ patients, which was equivalent to that of anti-EGFR antibodies with irinotecan. Further prospective longitudinal studies are needed.

\section{Acknowledgments}

We would like to thank staff in the Sun Yat-sen University Cancer Center for their work on treating patients. This article was supported by a grant from the Medical Research Fund 
in Guangdong Province, China (A2015413, C2017058) and National Natural Science Foundation of China (81602140).

\section{Disclosure}

The authors report no conflicts of interest in this work.

\section{References}

1. Torre LA, Bray F, Siegel RL, Ferlay J, Lortet-Tieulent J, Jemal A. Global cancer statistics, 2012. CA Cancer J Clin. 2015;65(2):87-108.

2. Grothey A, Van Cutsem E, Sobrero A, et al; CORRECT Study Group. Regorafenib monotherapy for previously treated metastatic colorectal cancer (CORRECT): an international, multicentre, randomised, placebo-controlled, phase 3 trial. Lancet. 2013;381(9863):303-312.

3. Van Cutsem E, Lenz HJ, Köhne CH, et al. Fluorouracil, leucovorin, and irinotecan plus cetuximab treatment and RAS mutations in colorectal cancer. J Clin Oncol. 2015;33(7):692-700.

4. Grothey A, Hedrick EE, Mass RD, et al. Response-independent survival benefit in metastatic colorectal cancer: a comparative analysis of N9741 and AVF2107. J Clin Oncol. 2008;26(2):183-189.

5. Modest DP, Ricard I, Heinemann V, et al. Outcome according to KRAS-, NRAS- and BRAF-mutation as well as KRAS mutation variants: pooled analysis of five randomized trials in metastatic colorectal cancer by the AIO colorectal cancer study group. Ann Oncol. 2016; 27(9):1746-1753.

6. Tampellini M, Di Maio M, Baratelli C, et al. Treatment of patients with metastatic colorectal cancer in a real-world scenario: probability of receiving second and further lines of therapy and description of clinical benefit. Clin Colorectal Cancer. 2017;16(4):372-376.

7. Heinemann V, von Weikersthal LF, Decker T, et al. FOLFIRI plus cetuximab versus FOLFIRI plus bevacizumab as first-line treatment for patients with metastatic colorectal cancer (FIRE-3): a randomised, open-label, phase 3 trial. Lancet Oncol. 2014;15(10):1065-1075.

8. NCCN Practice Guidelines for Colon Cancer (Version 2, 2017). Available from: https://www.nccn.org/professionals/physician_gls/pdf/colon. pdf. Accessed September 23, 2017.

9. Cunningham D, Humblet Y, Siena S, et al. cetuximab monotherapy and cetuximab plus irinotecan in irinotecan-refractory metastatic colorectal cancer. N Engl J Med. 2004;351(4):337-345.

10. Martín-Martorell P, Roselló S, Rodríguez-Braun E, Chirivella I, Bosch A, Cervantes A. Biweekly cetuximab and irinotecan in advanced colorectal cancer patients progressing after at least one previous line of chemotherapy: results of a phase II single institution trial. Br J Cancer. 2008; 99(3):455-458.

11. Mayer RJ, Van Cutsem E, Falcone A, et al; RECOURSE Study Group Randomized trial of TAS-102 for refractory metastatic colorectal cancer. N Engl J Med. 2015;372(20):1909-1919.
12. Giuliani J, Bonetti A. Epidermal growth factor inhibitors in first-line for metastatic colorectal cancer with ras wild-type: a perspective based on pharmacological costs. Expert Rev Pharmacoecon Outcomes Res. 2017; 17(3):243-248.

13. Le DT, Uram JN, Wang H, et al. PD-1 blockade in tumors with mismatch-repair deficiency. N Engl J Med. 2015;372:2509-2520.

14. Van Cutsem E, Köhne CH, Hitre E, et al. Cetuximab and chemotherapy as initial treatment for metastatic colorectal cancer. N Engl J Med. 2009; 360(14):1408-1417.

15. Pfeiffer P, Nielsen D, Bjerregaard J, Qvortrup C, Yilmaz M, Jensen B. Biweekly cetuximab and irinotecan as third-line therapy in patients with advanced colorectal cancer after failure to irinotecan, oxaliplatin and 5-fluorouracil. Ann Oncol. 2008;19(6):1141-1145.

16. Costa T, Nuñez J, Felismino T, Boente L, Mello C. REOX: Evaluation of the Efficacy of Retreatment With an Oxaliplatin-containing Regimen in Metastatic Colorectal Cancer: A Retrospective Single-center Study. Clin Colorectal Cancer. 2017;16(4):316-323.

17. Santini D, Vincenzi B, La Cesa A, et al. Continuous infusion of oxaliplatin plus chronomodulated capecitabine in 5-fluorouracil- and irinotecanresistant advanced colorectal cancer patients. Oncology. 2005;69(1): 27-34.

18. Suenaga M, Mizunuma N, Matsusaka S, et al. Phase II study of reintroduction of oxaliplatin for advanced colorectal cancer in patients previously treated with oxaliplatin and irinotecan: RE-OPEN study. Drug Des Devel Ther. 2015;9:3099-3108.

19. Kemeny N, Garay CA, Gurtler J, et al. Randomized multicenter phase II trial of bolus plus infusional fluorouracil/leucovorin compared with fluorouracil/leucovorin plus oxaliplatin as third-line treatment of patients with advanced colorectal cancer. J Clin Oncol. 2004;22(23): 4753-4761.

20. Chau I, Webb A, Cunningham D, et al. Oxaliplatin and protracted venous infusion of 5-fluorouracil in patients with advanced or relapsed 5-fluorouracil pretreated colorectal cancer. Br J Cancer. 2001;85(9): 1258-1264.

21. Luppi G, Zanelli F, Di Stasi A, Bertolini F, Pifferi M. Folfox2 regimen in heavily pretreated patients with advanced colorectal cancer. Tumori. 2002;88(4):270-272.

22. Martoni A, Mini E, Pinto C, et al. Oxaliplatin and protracted continuous 5-fluorouracil infusion in patients with pretreated advanced colorectal carcinoma. Ann Oncol. 2001;12(4):519-524.

23. Naing A, Kurzrock R. Chemotherapy resistance and retreatment: a dogma revisited. Clin Colorectal Cancer. 2010;9(2):E1-E4.

24. Tejpar S, Prenen H, Mazzone M. Overcoming resistance to antiangiogenic therapies. Oncologist. 2012;17(8):1039-1050.

25. Briani C, Argyriou AA, Izquierdo C, et al. Long-term course of oxaliplatin-induced polyneuropathy: a prospective 2-year follow-up study. J Peripher Nerv Syst. 2014;19(4):299-306.
OncoTargets and Therapy

\section{Publish your work in this journal}

OncoTargets and Therapy is an international, peer-reviewed, open access journal focusing on the pathological basis of all cancers, potential targets for therapy and treatment protocols employed to improve the management of cancer patients. The journal also focuses on the impact of management programs and new therapeutic agents and protocols on

\section{Dovepress}

patient perspectives such as quality of life, adherence and satisfaction. The manuscript management system is completely online and includes a very quick and fair peer-review system, which is all easy to use. Visit http://www.dovepress.com/testimonials.php to read real quotes from published authors. 